\title{
Diagnostic implications of CZT SPECT and impact of CT attenuation correction
}

\author{
Andrew Peters, MD, ${ }^{\mathrm{a}}$ Jeevan Kumar, $M D,{ }^{\mathrm{a}}$ and Pravin V. Patil, $\mathrm{MD}^{\mathrm{a}}$ \\ a Section of Cardiology, Department of Medicine, Lewis Katz School of Medicine at Temple \\ University, Philadelphia, PA
}

Received Jun 1, 2017; accepted Jun 2, 2017

doi: 10.1007/s12350-017-0961-5

\section{See related article, pp. 236-245}

In the current issue, Kennedy et al. ${ }^{1}$ further validate cadmium-zinc-telluride (CZT) SPECT, including the use of customized databases for automated perfusion scoring. They also demonstrate the utility of CT attenuation correction to reduce false positives and improve diagnostic accuracy. They examined three groups of patients, performing a comparison of CZT and conventional SPECT, establishing a customized database for automated perfusion scoring with CZT, and demonstrating the impact of CT attenuation correction (CTAC) in CZT SPECT. While there have been many studies comparing conventional SPECT to invasive angiography and CZT SPECT to invasive angiography, not many have directly compared them in the same population.

\section{CZT VERSUS CONVENTIONAL SPECT}

Single-photon emission computed tomography (SPECT) myocardial perfusion imaging (MPI) is an important non-invasive tool for the evaluation of coronary artery disease (CAD). The relationship of functional ischemia on non-invasive testing and angiographic disease on invasive angiography has been debated heavily, but strong evidence supports the use of stress MPI for diagnosis and prognosis of CAD in certain patients. SPECT MPI has its limitations, such as

\footnotetext{
Funding This research received no specific Grant from any funding agency in the public, commercial, or not-for-profit sectors.

Reprint requests: Pravin V. Patil MD, Section of Cardiology, Department of Medicine, Lewis Katz School of Medicine at Temple University, 9th Floor Parkinson Pavilion, 3401 N. Broad Street, Philadelphia, PA 19140; pravin.patil@tuhs.temple.edu

J Nucl Cardiol 2019;26:246-9.

$1071-3581 / \$ 34.00$

Copyright (C) 2017 American Society of Nuclear Cardiology.
}

attenuation artifact and image resolution, which can affect the predictive value and impact the clinical utility.

Conventional SPECT consists of a scintillation detector of sodium iodide (NaI) crystals. These cameras have been validated with good sensitivity and specificity leading to widespread clinical use. Conventional SPECT MPI has a sensitivity ranging between 0.82 and 0.91 , and specificity ranging between 0.70 and $0.90 .^{2}$ During the first decade of the twenty first century, CZT SPECT systems emerged promising ultrafast acquisition, dose reduction, and similar diagnostic accuracy. The direct conversion of gamma radiation into signal by the semiconductor ensured improved image fidelity.

CZT SPECT has been discussed extensively in the literature since cameras have a higher sensitivity compared to conventional cameras and reduced imaging time. ${ }^{2}$ Many of these studies have assessed the diagnostic performance of MPI with CZT cameras (Table 1). ${ }^{1,3-8}$ Further, in a meta-analysis by Nudi et al., the sensitivity of CZT SPECT was found to be range between 0.78 and 0.89 and the specificity ranged from 0.62 to $0.76 .{ }^{9}$ While the sensitivity is comparable to prior studies, the specificity is lower. Neill et al. further emphasized this with a direct comparison of CZT to conventional SPECT MPI in patients with correlative invasive angiography. CZT SPECT again had superior sensitivity but decreased specificity when compared to conventional SPECT. ${ }^{8}$ It is speculated that the lower specificity of CZT imaging is due to the practice of performing upright imaging, which may enhance patient comfort but results in more abdominal attenuation artifact, as well as the clinical tendency for use in an obese patient subgroup.

Kennedy et al. ${ }^{1}$ demonstrate a reasonable correlation between CZT and conventional NaI scanners. Their data are in agreement with prior studies that indicate CZT could be associated with lower specificity and a higher number of false positives. Further, they provide low-intermediate risk patient data that support the use of 
Table 1. Comparison of CZT and conventional SPECT

\begin{tabular}{lllllll}
\hline \multicolumn{1}{c}{ Author } & \multicolumn{1}{c}{ Type } & $\boldsymbol{n}$ & \multicolumn{1}{c}{ SRS } & \multicolumn{1}{c}{ SSS } & $\begin{array}{c}\text { Sensitivity } \\
\text { (\%) }\end{array}$ & $\begin{array}{c}\text { Specificity } \\
\text { (\%) }\end{array}$ \\
\hline Kennedy et al. $^{1}$ & CZT & 80 & $2.91 \pm 5.06$ & $5.56 \pm 5.95$ & N/A & N/A \\
& $\begin{array}{l}\text { CZT (Custom } \\
\text { Database) }\end{array}$ & & $1.78 \pm 4.47$ & $3.64 \pm 5.41$ & & \\
& Nal & & $1.95 \pm 4.15$ & $3.76 \pm 5.02$ & & \\
Barone-Rochette et al. $^{3}$ & CZT & 214 & $3.1 \pm 4.3$ & $8.0 \pm 4.9$ & 94 & 50 \\
Pourmoghaddas et al. $^{4}$ & CZT & 108 & $7.6 \pm 3.3$ & $8.33 \pm 2.97$ & N/A & N/A \\
& NaI & & $8.8 \pm 2.9$ & $9.62 \pm 2.28$ & & 78 \\
Gimelli et al. $^{5}$ & CZT & 248 & $2 \pm 2$ & $9 \pm 5$ & 89 & 70 \\
Nishiyama et al. $^{6}$ & CZT & 276 & $5 \pm 8$ & $9 \pm 8$ & 87 & 77 \\
Gimelli et al. $^{7}$ & CZT & 137 & $3 \pm 3$ & $10 \pm 5$ & 95 & 57 \\
Neill et al. $^{8}$ & CZT & 53 & N/A & N/A & 89 & 77 \\
& NaI & & & & 86 & 79 \\
\hline
\end{tabular}

a separate custom database for CZT automated perfusion scoring, which potentially could improve specificity. It will be important for further research to be performed with a higher risk cohort. The authors also incorporated CTAC into their analysis, validating its use.

\section{ROLE FOR CT ATTENUATION CORRECTION}

SPECT MPI is susceptible to attenuation artifacts from tissues such as breast, diaphragm, abdomen, and lateral chest wall that reduce specificity. ${ }^{10,11}$ Numerous techniques have been shown to reduce attenuation artifact including breast binding, prone imaging, and attenuation maps created by external sources. External fixed line source attenuation correction (AC) using most frequently gadolinium-153 but also cobalt-57, barium-133, americium-241, and technetium-99m has been used. ${ }^{12}$ Nishiyama et al. demonstrated a technique involving combined supine and prone SPECT acquisition could reduce the false-positive rate associated with supine image acquisition. ${ }^{6}$ Supine and upright imaging with CZT in obese patients has also demonstrated diagnostic accuracy for CAD. ${ }^{13,14}$ Although these techniques have been used, more recently hybrid SPECT/CT systems allow for high-quality attenuation maps due to improved signal-to-noise ratio and better resolution. ${ }^{15}$

CTAC for conventional SPECT imaging has been validated to improve diagnostic accuracy and risk stratification compared with non-attenuation (NAC) corrected imaging. ${ }^{16-18}$ CTAC has the advantage over line source $\mathrm{AC}$ in that there is higher photon flux, minimizing the interaction with the SPECT radionuclide without decay, and overall reduced scan times.
CZT SPECT is becoming more prolific in clinic practice due to the advantages of improved image fidelity and shorter scan times compared with $\mathrm{NaI}$ SPECT. ${ }^{19}$ Despite these apparent benefits, CZT is still susceptible to attenuation artifact. Liu et al. used an anthropomorphic torso phantom and found that CZT had higher myocardial count sensitivity, better signal-tonoise ratio, and superior uniformity of overall myocardial tracer uptake compared to NaI; however, CZT was more susceptible to inferior and inferolateral attenuation with lower normalized uptake compared to the $\mathrm{NaI}$ camera in the anthropomorphic torso model to simulate a male patient. ${ }^{20}$ Similarly, Herzog et al. reported predominant artifacts in the right coronary artery territory in males. ${ }^{21}$ In contrast, CZT performed better than the NaI camera in imaging anterior defects. ${ }^{20}$ This phenomenon may be explained by the design of the CZT camera, since the pinhole collimator is closest to the linear source in the anterior position and furthest in the right lateral position.

Multiple studies have assessed the diagnostic performance of CZT SPECT with CTAC, using invasive coronary angiography as a comparator. Van Dijk et al. retrospectively looked at 107 patients with low to intermediate probability of CAD that underwent stress only MPI. Diagnostic confidence increased using CTAC as $80 \%$ of scans were interpreted as normal or abnormal, rather than equivocal, versus $57 \%$ of scans without $\mathrm{AC}$ $(P<0.001) .{ }^{22}$ Caobelli et al. looked at 60 patients, 44 of which underwent coronary angiography, to compare the performance of CZT with and without CTAC. Interestingly, the authors found significantly improved diagnostic accuracy in the RCA territory $(P=0.002)$ but not in the $\operatorname{LAD}(P=0.35)$ or $\operatorname{LCx}(P=0.08)$ 


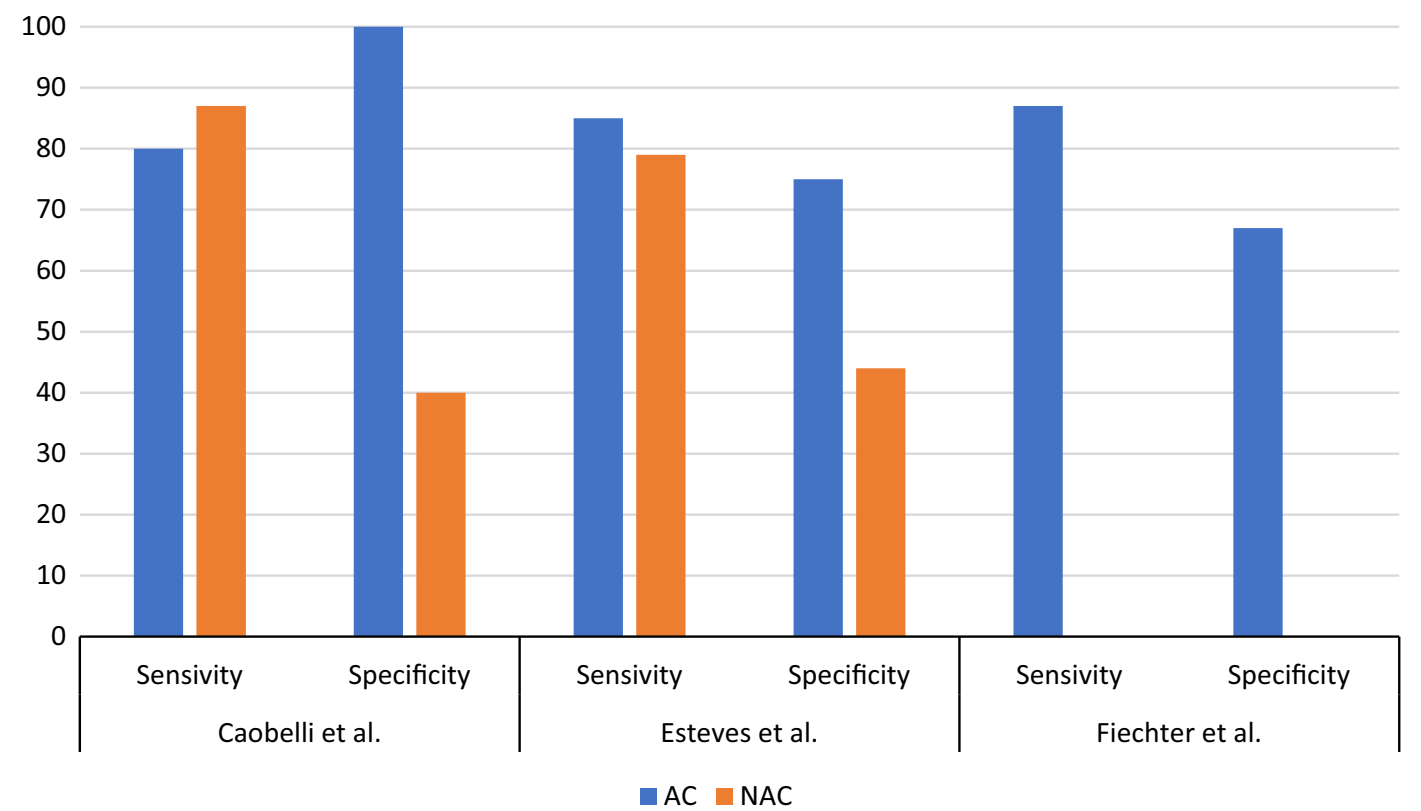

Figure 1. Effect of CT attenuation correction on the diagnostic accuracy of CZT SPECT.

territories. ${ }^{23}$ Esteves et al. investigated a subset of 55 patients that underwent coronary angiography within 2 months of CZT SPECT. In their primary analysis, there was a non-significant difference in specificity for obstructive CAD between CTAC and NAC $(75 \%$ versus $44 \% ; P=0.15) .{ }^{24}$ However, in a secondary analysis, the authors found that with quantitative analysis for all three vascular territories, NAC specificity was lower than CTAC (63\% versus $78 \%, P=0.02$ ) Finally, Fietcher et al. looked at 66 patients that underwent CZT SPECT/CT and catheterization within 3 months. The authors reported that with visual analysis the sensitivity/specificity of CZT SPECT was $87 \% / 67 \%$ and that with semi-automated analysis the sensitivity/ specificity was $74 \% / 67 \% .^{25}$ Overall, these studies reported high sensitivities and reasonable specificities (Figure 1); ${ }^{23-25}$ however, the number of patients in each study was limited.

Kennedy et al. ${ }^{1}$ add to the limited body of literature on the use of CTAC in CZT SPECT. They found that AC significantly decreased the SSS and SRS in men when compared with NAC, but not in women. This is in agreement with the current literature and highlights that CZT is more susceptible to inferior attenuation artifact that can masquerade as an RCA territory perfusion abnormality. Although invasive angiographic correlation was not performed by the authors, their data suggest CTAC may lead to a reduction in unnecessary invasive procedures.

\section{Disclosure}

The authors report no conflict of interest.

\section{References}

1. Kennedy JA, Brodov Y, Weinstein AL, Israel O, Frenkel A. The effect of CT-based attenuation correction on the automatic perfusion score of myocardial perfusion imaging using a dedicated cardiac solid-state CZT SPECT/CT. J Nucl Cardiol. 2017. doi:10.1007/s12350-017-0905-0.

2. Agostini D, Marie P, Ben-Haim S, et al. Performance of cardiac cadmium-zinc-telluride gamma camera imaging in coronary artery disease: A review from the cardiovascular committee of the european association of nuclear medicine (EANM). Eur J Nucl Med Mol Imaging. 2016;43(13):2423-32.

3. Barone-Rochette G, Leclere M, Calizzano A, et al. Stress thallium-201/rest technetium-99m sequential dual-isotope high-speed myocardial perfusion imaging validation versus invasive coronary angiography. J Nucl Cardiol. 2015;22(3):513-22.

4. Pourmoghaddas A, Vanderwerf K, Ruddy T, Glenn Wells R. Scatter correction improves concordance in SPECT MPI with a dedicated cardiac SPECT solid-state camera. J Nucl Cardiol. 2015;22(2):334-43.

5. Gimelli A, Bottai M, Genovesi D, et al. High diagnostic accuracy of low-dose gated-SPECT with solid-state ultrafast detectors: Preliminary clinical results. Eur J Nucl Med Mol Imaging. 2012;39(1):83-90.

6. Nishiyama Y, Miyagawa M, Kawaguchi N, et al. Combined supine and prone myocardial perfusion single-photon emission computed tomography with a cadmium zinc telluride camera for detection of coronary artery disease. Circ J. 2014;78(5):1169-75.

7. Gimelli A, Bottai M, Quaranta A, Giorgetti A, Genovesi D, Marzullo P. Gender differences in the evaluation of coronary 
artery disease with a cadmium-zinc telluride camera. Eur J Nucl Med Mol Imaging. 2013;40(10):1542-8.

8. Neill J, Prvulovich E, Fish M, et al. Initial multicentre experience of high-speed myocardial perfusion imaging: Comparison between high-speed and conventional single-photon emission computed tomography with angiographic validation. Eur J Nucl Med Mol Imaging. 2013;40(7):1084-94.

9. Nudi F, Iskandrian AE, Schillaci O, Peruzzi M, Frati G, BiondiZoccai G. Diagnostic accuracy of myocardial perfusion imaging with CZT technology. JACC: Cardiovase Imaging. 2017. doi: 10.1016/j.jcmg.2016.10.023.

10. Dunn RF, Wolff L, Wagner S, Botvinick EH. The inconsistent pattern of thallium defects: A clue to the false positive perfusion scintigram. Am J Cardiol. 1981;48(2):224-32.

11. DePuey EG, Garcia EV. Optimal specificity of thallium-201 SPECT through recognition of imaging artifacts. J Nucl Med. 1989;30(4):441.

12. Hendel RC, Corbett JR, Cullom SJ, DePuey EG, Garcia EV, Bateman TM. The value and practice of attenuation correction for myocardial perfusion SPECT imaging: A joint position statement from the american society of nuclear cardiology and the society of nuclear medicine. J Nucl Cardiol. 2002;9(1):135-43.

13. Nakazato R, Slomka P, Fish M, et al. Quantitative high-efficiency cadmium-zinc-telluride SPECT with dedicated parallel-hole collimation system in obese patients: Results of a multi-center study. J Nucl Cardiol. 2015;22(2):266-75.

14. Ben-Haim S, Almukhailed O, Neill J, et al. Clinical value of supine and upright myocardial perfusion imaging in obese patients using the D-SPECT camera. J Nucl Cardiol. 2014;21(3):478-85.

15. Goetze S, Brown TL, Lavely WC, Zhang Z, Bengel FM. Attenuation correction in myocardial perfusion SPECT/CT: Effects of misregistration and value of reregistration. $\mathrm{J}$ Nucl Med. 2007;48(7):1090-5.

16. Masood Y, Liu Y, DePuey G, et al. Clinical validation of SPECT attenuation correction using $\mathrm{X}$-ray computed tomography-derived attenuation maps: Multicenter clinical trial with angiographic correlation. J Nucl Cardiol. 2005;12(6):676-86.
17. Schepis $\mathrm{T}$, Gaemperli $\mathrm{O}$, Koepfli $\mathrm{P}$, et al. Use of coronary calcium score scans from stand-alone multislice computed tomography for attenuation correction of myocardial perfusion SPECT. Eur J Nucl Med Mol Imaging. 2007;34(1):11-9.

18. Pazhenkottil AP, Ghadri J, Nkoulou RN, et al. Improved outcome prediction by SPECT myocardial perfusion imaging after CT attenuation correction. J Nucl Med. 2011;52(2):196-200.

19. Mouden M, Timmer J, Ottervanger JP, Reiffers S, Oostdijk A, Knollema S, Jager P. Impact of a new ultrafast CZT SPEC camera for myocardial perfusion imaging: Fever equivocal results and lower radiation dose. Eur J Med Mol Imaging. 2012;39:1048-55.

20. Liu C, Cheng J, Chen Y, Huang Y, Yen R. A performance comparison of novel cadmium-zinc-telluride camera and conventional SPECT/CT using anthropomorphic torso phantom and water bags to simulate soft tissue and breast attenuation. Ann Nucl Med. 2015;29(4):342-50.

21. Herzog B, Buechel R, Husmann L, Pazhenkottil A, Burger I, Wolfrum M, Nkoulous R, Valenta I, Ghadri J, Treyer V, Kaufmann P. Validation of CT attenuation correction for high-speed myocardial perfusion imaging using a novel cadmium-zinc-telluride detector technique. J Nucl Med. 2010;51(10):1539-44.

22. van Dijk JD, Mouden M, Ottervanger JP, et al. Value of attenuation correction in stress-only myocardial perfusion imaging using CZT-SPECT. J Nucl Cardiol. 2017;24(2):395-401.

23. Caobelli F, Akin M, Thackeray JT, et al. Diagnostic accuracy of cadmium-zinc-telluride-based myocardial perfusion SPECT: Impact of attenuation correction using a co-registered external computed tomography. Eur Heart $\mathrm{J}$ Cardiovasc Imaging. 2016;17(9):1036-43.

24. Esteves F, Galt J, Folks R, Verdes L, Garcia E. Diagnostic performance of low-dose rest/stress tc-99m tetrofosmin myocardial perfusion SPECT using the 530c CZT camera: Quantitative vs visual analysis. J Nucl Cardiol. 2014;21(1):158-65.

25. Fiechter M, Gebhard C, Fuchs TA, et al. Cadmium-zinc-telluride myocardial perfusion imaging in obese patients. J Nucl Med. 2012;53(9):1401-6. 